\title{
Structure and spectrum of anisotropically confined two-dimensional clusters with logarithmic interaction
}

\author{
S. W. S. Apolinario, ${ }^{*}$ B. Partoens, ${ }^{\dagger}$ and F. M. Peeters ${ }^{\ddagger}$ \\ Departement Fysica, Universiteit Antwerpen (Campus Middelheim), Groenenborgerlaan 171, B-2020 Antwerpen, Belgium
}

(Received 18 April 2005; published 18 October 2005)

\begin{abstract}
We studied the structural and spectral properties of a classical system consisting of a finite number of particles, moving in two dimensions, and interacting through a repulsive logarithmic potential and held together by an anisotropic harmonic potential. Increasing the anisotropy of the confinement potential can drive the system from a two-dimensional (2D) to a one-dimensional (1D) configuration. This change occurs through a sequence of structural transitions of first and second order which are reflected in the normal mode frequencies. Our results of the ground state configurations are compared with recent experiments and we obtained a satisfactory agreement. The transition from the 1D line structure to the 2D structure occurs through a zigzag transition which is of second order. We found analytical expressions for the eigenfrequencies before the zigzag transition, which allowed us to obtain an analytical expression for the anisotropy parameter at which the zigzag transition occurs as a function of the number of particles in the system.
\end{abstract}

DOI: 10.1103/PhysRevE.72.046122

PACS number(s): 64.60.-i, 63.22.+m

\section{INTRODUCTION}

Due to strong Coulomb repulsion, an electron gas at low temperature and density undergoes a phase transition to a crystalline state called the Wigner crystal, as predicted theoretically by Wigner in 1934 [1]. The Wigner crystal is one of the possible ground states of a strongly correlated electron system. Wigner crystallization was first observed in an electron gas confined above the surface of liquid helium by Grimes and Adams [2]. Several theoretical and experimental works have considered the properties of a finite number of particles interacting through a repulsive interparticle interaction potential and kept together by an external confinement potential. Typical experimental realizations of such twodimensional (2D) systems include electrons on the surface of liquid helium [2], electrons in quantum dots [3], colloidal suspensions [4], and confined plasma crystals [5]. These systems and their configurations have been observed experimentally, and are important in solid-state physics and plasma physics as well as in atomic physics.

Not only clusters with particles interacting through the Coulomb potential are investigated but also clusters with logarithmic interaction has attracted attention because they correspond with real experimental systems. Vortices in a film of liquid helium interact through a logarithmic potential [6], and also a low concentration of vortices in a type II superconducting 2D film interact through a logarithmic potential [7]. The logarithmic interaction between vortices was used to study the stable vortex configurations in a disk shaped superconductor $[8,9]$. Recently, it was shown $[10,11]$ that the experimental configurations of charged metallic balls on the bottom of a plane horizontal capacitor were better described by a logarithmic interparticle potential $[12,13]$ than a Coulomb interaction potential [14-16].

\footnotetext{
*Electronic address: sergio.apolinario@ua.ac.be

†Electronic address: bart.partoens@ua.ac.be

‡Electronic address: francois.peeters@ua.ac.be
}

An anisotropic confinement potential is considered in the experiment of Ref. [11] which inspired us to investigate the effect of the symmetry of the confinement on the configuration of the particles. The experimental setup [11] consists of charged metallic balls on the bottom electrode of a plane horizontal capacitor, the upper electrode being a transparent conducting glass. To get the ball charged, a potential $V_{e}$ of $1000 \mathrm{~V}$ is applied to this capacitor. And in order to confine the balls, another potential $V_{c}$ of $2000 \mathrm{~V}$ is applied to a metallic elliptic frame intercalated between the two capacitor electrodes. The experimental cell is fixed on a plate that leans on three independent loudspeakers. Initially the cell is strongly shaken and the system is liquid at this initial stage. Consecutively, a sequence of appropriate annealing introduced by loudspeakers vibrations induces the system to a stable configuration. Throughout the experiment, images of array balls are recorded in real time using a charge-coupled device camera onto a VHS videocassette recorder. By analyzing these records and measuring the time spent in each observed configuration, the "ground state configuration" was retained as the most frequently observed state. It was shown that the asymmetry of the confinement induces various rearrangements of the particles, according to their number and the asymmetry of the potential. These rearrangements consist of a spatial cluster orientation and intershell particle exchanges. They also found some metastable configurations and well defined triangular structures. In our model the particles interact through a logarithmic interaction potential as suggested by the experiment. We will show in this paper that we could reobtain all configurations presented in Ref. [11]. We classified the transitions as first and second order which, respectively, exhibit a discontinuity in the first and second derivative of the energy as a function of the anisotropy of the confinement. Also we show that these transitions affect the eigenfrequency spectrum of the system. Reference [17] recently investigated a similar system of logarithmically interacting particles but with a circular confinement. Here we will stress the effects due to anisotropy. Previously Ref. [18] con- 
sidered Coulomb interacting particles in an elliptic confinement potential, but at that time no experimental results were available. Furthermore, here we found an analytical expression for all the eigenfrequencies before the zigzag transition. This allowed us to find an expression for the anisotropy parameter at which the zigzag transition occurs as a function of the number of particles in the system.

The paper is organized as follows. In Sec. II we present our model system. In Sec. III we obtain the ground state properties and structural transitions and compare them with the experimental results of Ref. [11]. The dynamical properties before the zigzag transition and some eigenfrequency modes which are independently of the number of particles are investigated in Sec. IV. Finally we present our conclusions in Sec. V.

\section{MODEL AND NUMERICAL APPROACH}

We study a 2D model system of $N$ equally charged particles in an elliptic confinement potential and interacting through a repulsive logarithmic potential. The Hamiltonian of the system is given by

$$
H=\sum_{i=1}^{N} \frac{1}{2} m\left(\omega_{0 x}^{2} x_{i}^{2}+\omega_{0 y}^{2} y_{i}^{2}\right)-\sum_{i>j}^{N} \beta \ln \left|\mathbf{r}_{i}-\mathbf{r}_{j}\right|
$$

where $m$ is the mass of the particle, $\mathbf{r}_{i}=\left(x_{i}, y_{i}\right)$ is the vector position of the $i$ th particle, $\beta$ gives the strength of the interaction, and $\omega_{0 x}$ and $\omega_{0 y}$ are, respectively, the confinement frequencies in the $x$ and $y$ directions. These frequencies are related by the expression $\omega_{0 x}=\sqrt{\alpha} \omega_{0 y}$ where $\alpha$ is the eccentricity of the elliptic confinement.

We can write the Hamiltonian (1) in a dimensionless form if we express the coordinates, energy, and temperature in the units $r_{0}=(2 \beta)^{1 / 2} m^{-1 / 2}, E_{0}=\beta$, and $T_{0}=\beta k_{B}^{-1}$, where $k_{B}$ is the Boltzmann constant. The dimensionless Hamiltonian is

$$
H=\sum_{i=1}^{N}\left(\alpha x_{i}^{2}+y_{i}^{2}\right)-\sum_{i>j}^{N} \ln \left|\mathbf{r}_{i}-\mathbf{r}_{j}\right| .
$$

All the results will be given in dimensionless units. The ground state configuration is the global minimum of the potential energy which is only a function of the number of charged particles $N$ and the eccentricity $\alpha$. Our numerical method to obtain the ground state configuration is based on the Monte Carlo simulation technique supplemented with the Newton method in order to increase the accuracy of the energy minimum [14]. By starting from different random initial configurations we are able to find all the possible stable (i.e., ground state and metastable) configurations. The eigenfrequencies are the square roots of the eigenvalues of the dynamical matrix

$$
H_{\alpha \beta, i j}=\left.\frac{\partial^{2} H}{\partial r_{\alpha, i} \partial r_{\beta}, j}\right|_{r_{\alpha, i}=r_{\alpha, i}^{n}}
$$

where $\left\{r_{\alpha, i}^{n} ; \alpha=x, y ; i=1, \ldots, N\right\}$ is the position of the particles in the ground state configuration.

\section{GROUND STATE CONFIGURATIONS AND STRUCTURAL TRANSITIONS}

At low temperature and for circular confinement the observed clusters are self-organized patterns constituted by "concentric" shells on which the particles are located. Changing eccentricity $\alpha$ from 1 to 0 , we can go from a circular potential to a $1 \mathrm{D}$ system where the concentric shell arrangements are broken and all particles are aligned along the $x$ axis. In this last limit the particles are infinitely far from each other since the system becomes unbound in the $x$ direction. In this section, we take the system of $N=6$ particles as an example to present some general properties of the ground state configurations and its configurational transition as a function of the anisotropy parameter $\alpha$. In particular, we give a detailed overview of first and second order transitions for this system. Next, we will analyze the experimental results of Ref. [11] which focused on small systems with $N=4,5,6$ and 9 particles, large systems with $N=16$ and 17 particles, and systems with some well defined triangular structures.

Changing the eccentricity can induce two types of transitions in the ground state configuration. One of the transitions involves an intershell particle exchange which can happen continuously or abruptly, as the eccentricity parameter is varied, characterizing, respectively, a second and first order transition. The other transition is just a continuous spatial cluster orientation that constitute a second order transition.

We can see these two types of transitions by varying the anisotropy parameter $\alpha$ from 1 to 0 for the $N=6$ system (see Fig. 1). With a decrease of $\alpha$ the ground state configuration passes through a first order transition involving an intershell particle exchange from configurations $(1,5)$ to $(6)$ at $\alpha=0.88$. These configurations are respectively shown in Figs. 2(i) and 2(j). Notice that this transition is not continuous in position space as we see in Figs. 1(b) and 1(c), which show, respectively, jumps in the coordinates $x$ and $y$ around $\alpha=0.88$. It is also accompanied by a discontinuity in the eigenfrequencies [see Fig. 1(a)].

The variation of elliptic eccentricity $\alpha$ induces also continuous configuration adjustments. These adjustments happen to reduce the system energy. At such continuous transitions one of the eigenfrequencies of the ground state goes to zero. This is the equivalent of the softening of a phonon mode in an infinite crystal structure. For $N=6$ particles such a softening occurs five times over the $\alpha=0 \rightarrow 1$ range. This occurs first at $\alpha=0.2$. In Fig. 2(a) we see that for $\alpha=0.19$ all particles are aligned along the $x$ axis forming a chainlike structure while for $\alpha=0.21$ [see Fig. 2(b)], just after the transition, the particles are no longer aligned with the $x$ axis. This transition is called the zigzag transition and is discussed in further detail in Sec. IV. Initially only the central particles in the line take part in the zigzag transition which can be verified by noticing the different slopes of the $y$ coordinate lines after $\alpha=0.2$ in Fig. 1(c). This behavior was also observed previously for the case of Coulomb interparticle interaction [18] and it is independent of the number of particles in the system. Notice that the oscillation amplitude of each particle decreases when going from the center of the system to the edge of the line configuration as shown in Fig. 2(a) by the length of the eigenvectors. 


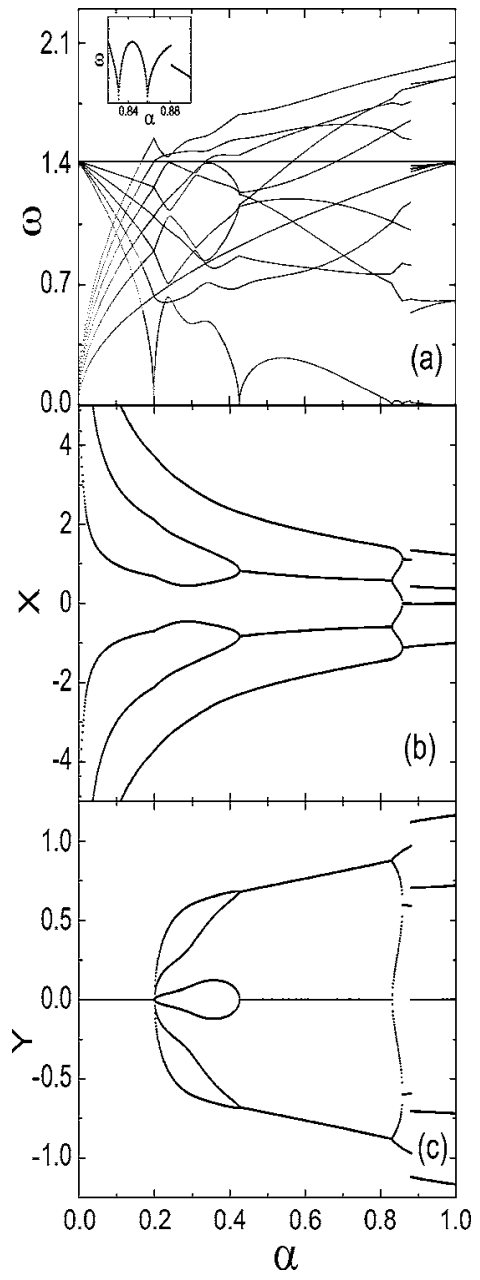

FIG. 1. (a) The mode spectrum as a function of the anisotropy parameter for a cluster of $N=6$ particles. (b) and (c) are, respectively, the $x$ and $y$ particle coordinates as a function of $\alpha$.

The eigenfrequency related to the rotational mode goes to zero at $\alpha=0.43$ and 0.84 which are related, respectively, with the alignment and disalignment of two particles with the $x$ axis as can be seen in Figs. 2(d) and 2(f), respectively. Also for $\alpha=0.86$ the rotational mode frequency is zero but now it is related to the alignment of two particles with the $y$ axis as we see in Fig. 2(h). Finally, for $\alpha=1$, the system exhibits a zero frequency rotational mode because of the rotational symmetry of the potential.

The experimental work in Ref. [11] showed ground state configurations for $N=4,5,6$, and 9 and values of $\alpha$ varying from 0.5 to 1 . We also present configurations related to these systems in Fig. 3. Note that the increase of the confinement asymmetry, i.e., decreasing of $\alpha$, results in intra- and/or intershell reorganization of the particles. For a one-shell arrangement, illustrated for $N=4$ and 5 particles, this reorganization is characterized by a particular orientation of the cluster with respect to the major axis of the elliptic confinement. Our results for the ground state configuration for $N=4$ and 5 are compatible with the experimental ones of Ref. [11] except for the configuration of $N=5$ at $\alpha=0.5$ where the configuration found in Ref. [11] corresponds to a saddle point in our model which can be seen in Fig. 4(a).

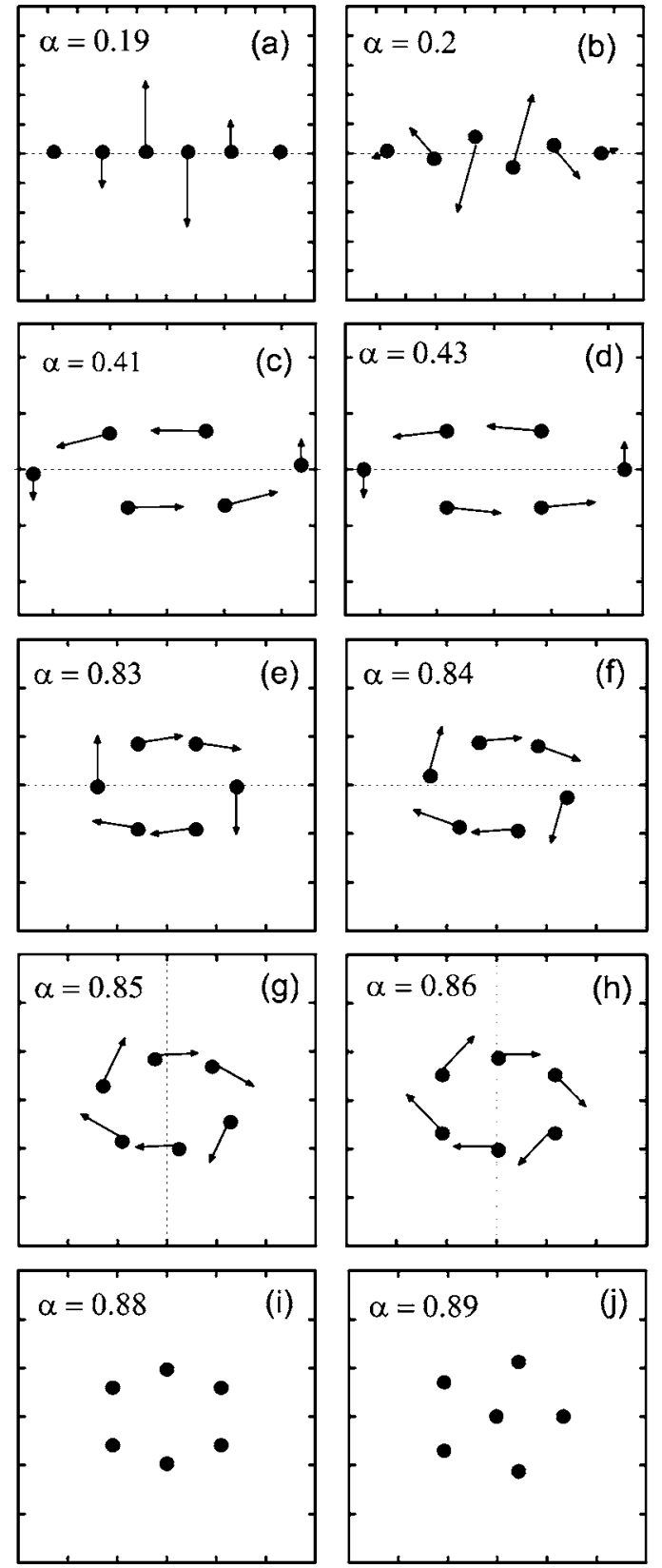

FIG. 2. Ground state configurations for different values of the eccentricity parameter $\alpha$. The eigenvectors corresponding to the lowest normal mode are indicated in (a)-(h). The scale is different in each figure, but the distance between the ticks is always one length unit.

This difference from the experimental result may be attributed to the fact that the difference in energy between both states is very small at $\alpha=0.5$, namely, $\Delta E / E<4.6 \%$.

For a two-shell structure, the asymmetric confinement can induce shell arrangements in the cluster. The system $N=6$ is an example in which a two-shell ground state configuration $(1,5)$ for circular confinement is transformed into a one-shell arrangement (6) when $\alpha$ decreases. This behavior is also observed for $N=7,8$, and 9 particles. On the other hand, the experimental paper [11] shows that the increase of the elliptic asymmetry induces, first, a transition from the $(1,8)$ configuration to the $(2,7)$ one, followed by another rearrange- 


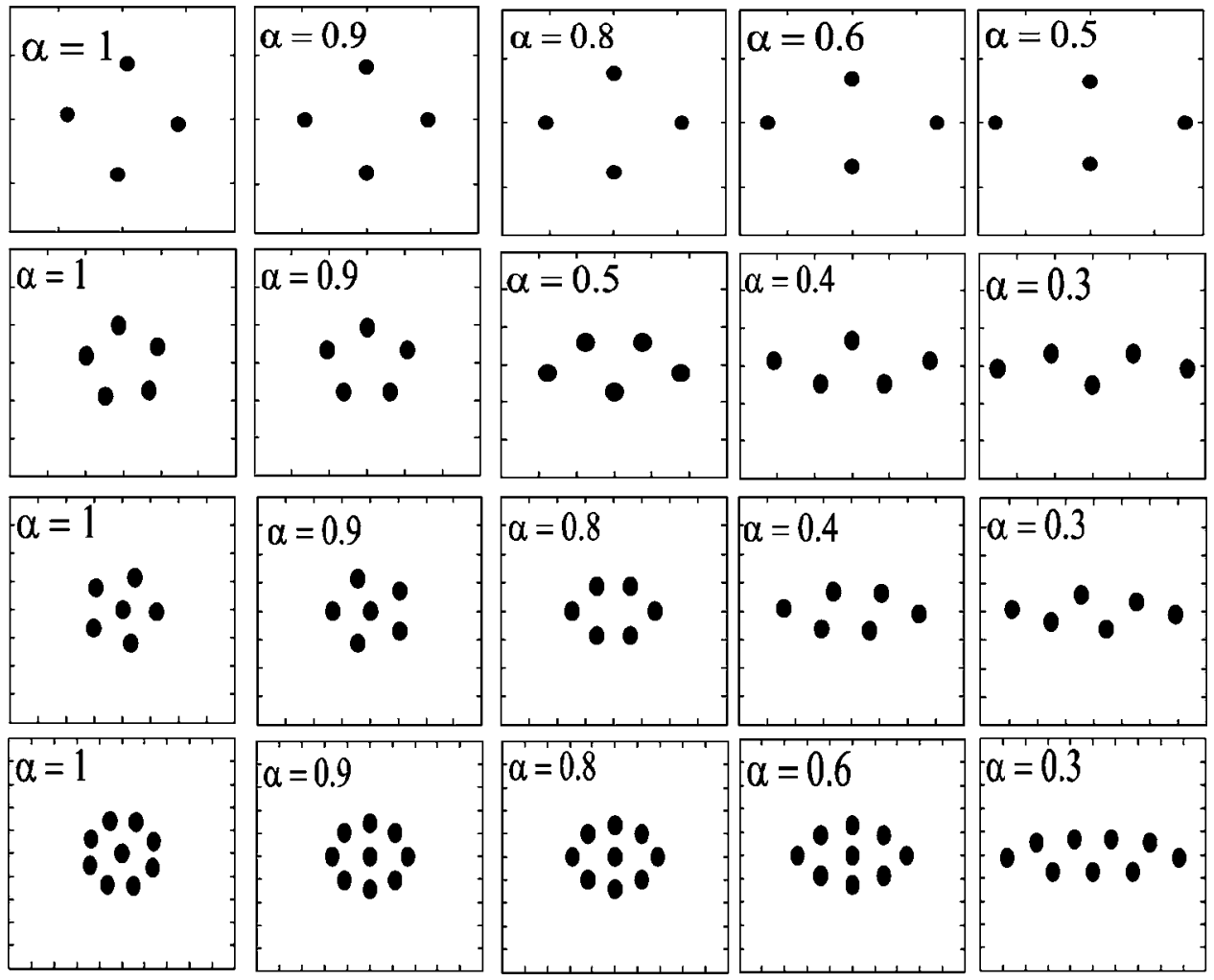

FIG. 3. The ground state configurations for different values of $N$ and $\alpha$ which correspond to the experimental results of Ref. [11]. The scale is different in each figure, but the distance between the ticks is always one length unit. ment which results in a return to the initial configuration $(1,8)$ for $\alpha=0.6$. However, in our model, the configuration $(2,7)$ which can be seen in Fig. 4(b) corresponds to a metastable state with energy very close to the energy of the ground state configuration $(1,8)$. We show the difference of energy between both configurations in Fig. 5. The energy difference between both states is very small in the region just around $\alpha=0.8$ where the configuration $(2,7)$ was found in the experimental work [11].

Now we turn our attention to larger systems where a well defined triangular structure are found (see Fig. 6). These configurations are in good agreement with the results obtained in Ref. [11]. These configurations are analogous to the "magic number structures" discussed in Ref. [14]. Independently if the circular system corresponds to a magic number one the elliptic potential can induce, for some $N$ and $\alpha$, a triangular array arrangement.

In Ref. [11] an experimental procedure was developed to investigate metastable configurations and they explored the influence of the anisotropy on the metastable states of the system. It was noted that the rules of transition between
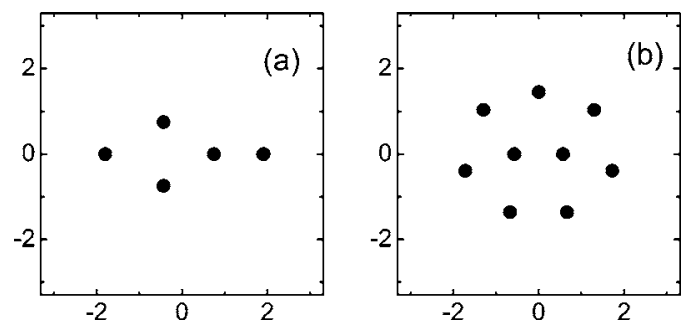

FIG. 4. (a) Saddle point configuration for a system with $N=5$ particles for $\alpha=0.5$. (b) Metastable state for $N=9$ and $\alpha=0.9$. ground state and metastable configurations appear differently for systems with different number of particles. To illustrate this topic, they considered the cases $N=16(\alpha=0.7)$ and 17 $(\alpha=0.6)$ which were also obtained using our theoretical method and they are shown in Fig. 7. The configuration of ground state (GS), metastable state (MS), and saddle point (SP) are, respectively, shown in Figs. 7(a), 7(b), and 7(c) for $N=16$ and Figs. 7(d), 7(e), and 7(f) for $N=17$. These configurations are classified slightly different in Ref. [11]. There the ground state and first metastable states obtained experimentally correspond, respectively, to the configurations shown in Figs. 7(a) and 7(c) for $N=16$ and in Figs. 7(d) and 7 (f) for $N=17$. The inner shell of these configurations plays a different transformation rule between the ground and first

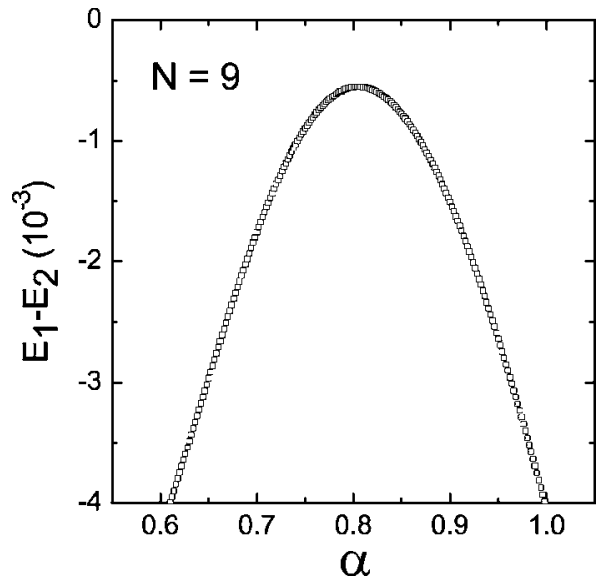

FIG. 5. Difference of energy between the ground state energy $E_{1}$ with configuration $(8,1)$ and the metastable energy $E_{2}$ with configuration $(7,2)$. 

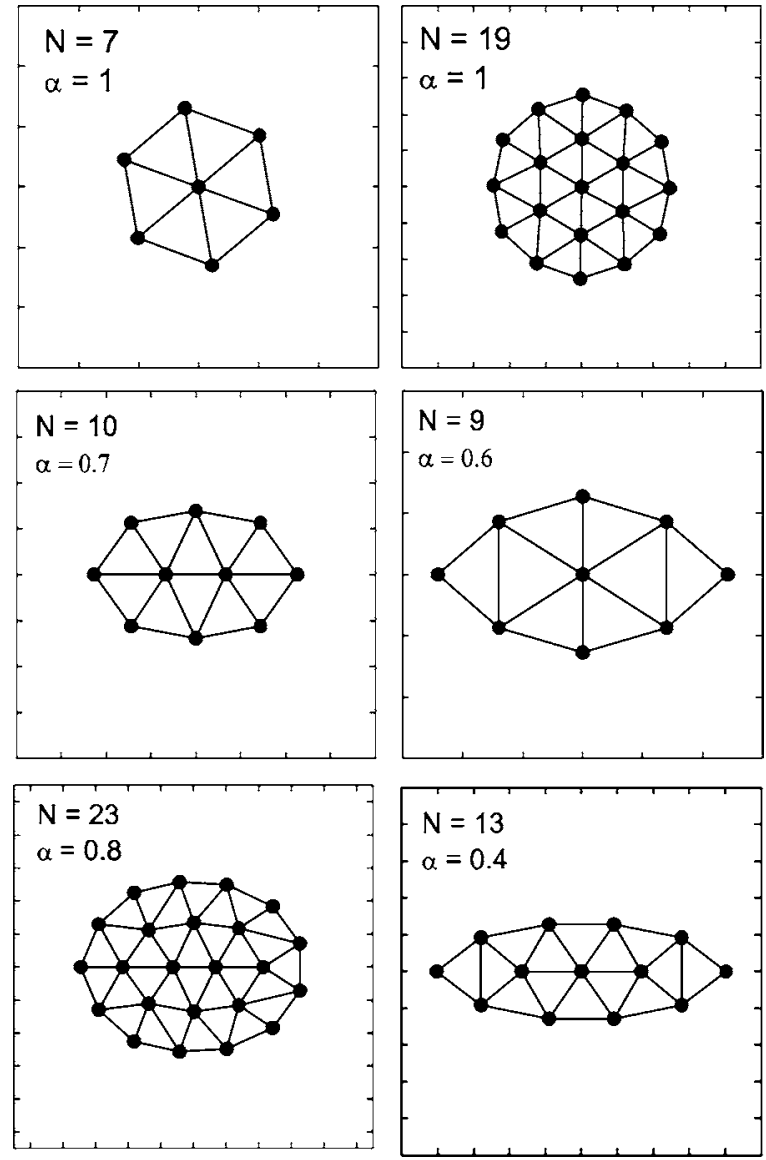

FIG. 6. Example of ground state configurations exhibiting a well defined triangular arrangement which is accentuated by the thin lines. The distance between the ticks is one length unit.

metastable states. Reference [11] noted that their ground state and metastable state for the system $N=16$ and $\alpha=0.7$ has, respectively, an inner shell corresponding to the ground state and metastable state configuration of a system with $N=5$ particles. While for the system with $N=17$ particles and $\alpha=0.6$ the opposite situation occurs, i.e., the inner shell of the ground state and metastable state configurations, corre-

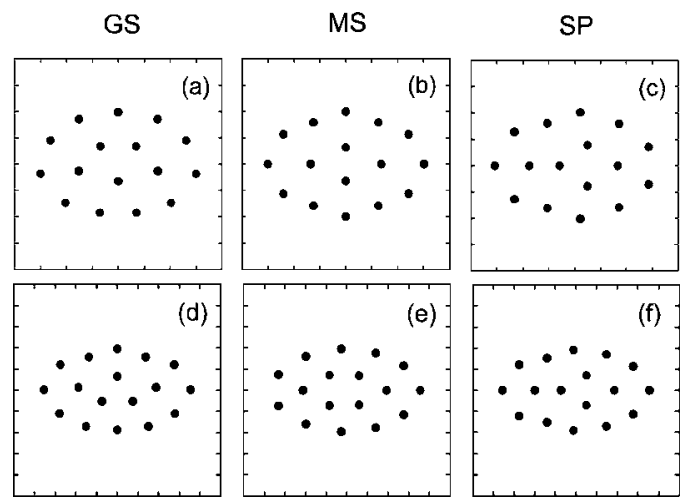

FIG. 7. Configurations for $N=16$ and 17 particles with, respectively, $\alpha=0.7$ and 0.6. From left to right the columns show the configurations corresponding to the ground state (GS), metastable state (MS), and saddle point (SP). The distance between the ticks is one length unit.

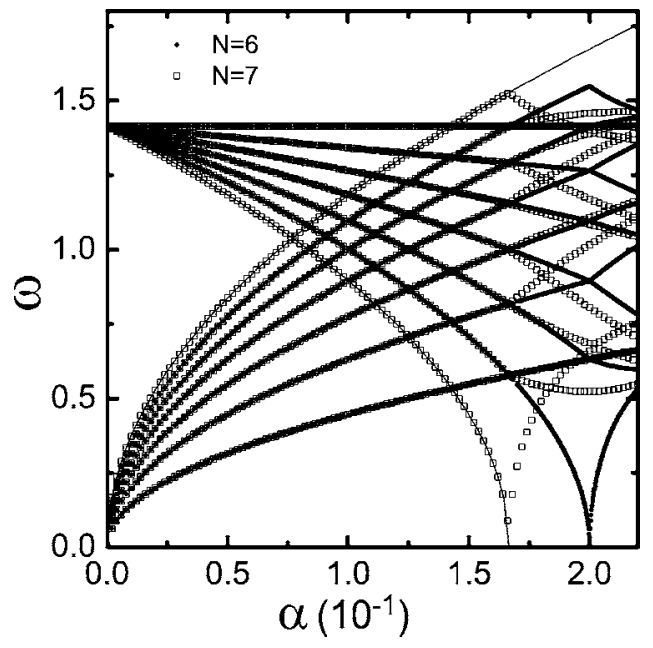

FIG. 8. The eigenmode spectrum as a function of the anisotropy parameter for a cluster of $N=6$ and 7 particles.

spond, respectively, to the metastable and ground state configurations of a system with $N=5$ particles. We noted that the shape structure of the outer shell played a different rule in the minimization of the configurational energy. For example, in the case of $N=16$ the cluster arrangement with a regular outer shell, Fig. 7(a), has a lower energy than the metastable configuration, Fig. 7(b), presenting an irregular outer shell. In contrast, the system with $N=17$ the configuration of lower energy Fig. 7(d), has an irregular outer shell while the cluster with regular outer shell, Fig. 7(e), has a larger energy. The energies of the GS, MS, and SP are, respectively, $E=-3.59426,-3.59294$, and -3.59175 for $N=16$ and $E=-4.42750,-4.42679$, and -4.42737 for $N=17$. We can see that the relative difference in energy between the configurations with the same number of particles are very small.

\section{DYNAMICAL PROPERTIES}

In this section we investigate in more detail the dependence of the eigenfrequency spectrum on the anisotropy of the confinement potential. The spectrum for the logarithmic interaction system was compared with the behavior of the eigenfrequencies found in Ref. [18] for a Coulomb system. We also investigate the center of mass mode and breathing mode eigenfrequency.

Before the zigzag transition we found that a system with $N+1$ particles has the same frequencies as a system with $N$ particle plus two extra frequencies. This is illustrated in Fig. 8 where the eigenfrequencies for the system with $N=6$ and 7 particles are shown, respectively, by closed and open dots. We can see that the closed dots completely coincide with the open dots before the zigzag transition which occurs at $\alpha=0.167$ for a system with $N=7$ particles. We found a general expression that fits all eigenfrequencies for a system of $N$ particles before the zigzag transition. These expressions are

$$
\begin{gathered}
\omega=\sqrt{2-2(n-1) \alpha}, \\
\omega=\sqrt{2 n \alpha},
\end{gathered}
$$




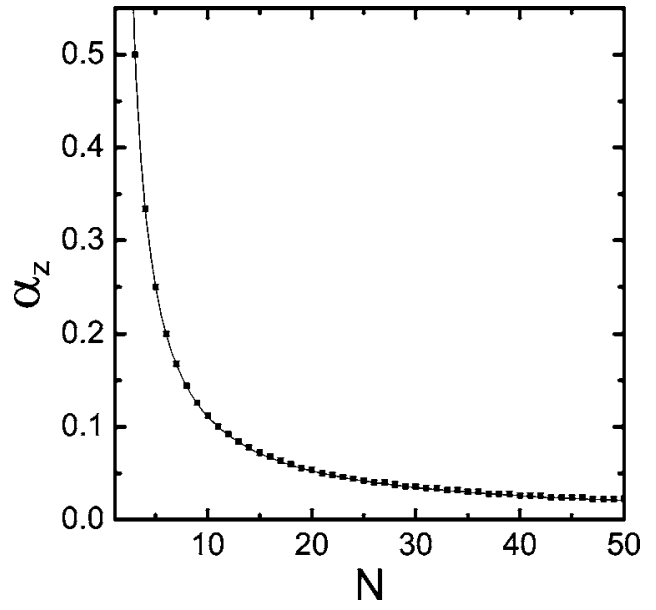

FIG. 9. The value of the anisotropy parameter at which the zigzag transition occurs as a function of the number of particles. The symbols are the results from our simulations and the curve is the expression $\alpha_{z}=1 /(N-1)$.

with $n=1, \ldots, N$. We show the fit for $n=7$ in Fig. 8. The zigzag transition in a system of $N$ particles occurs when the eigenfrequency given by the expression $\omega=\sqrt{2-2(N-1) \alpha}$ becomes zero. From this we can conclude that the zigzag transition occurs at $\alpha_{z}=1 /(N-1)$. In Fig. 9 we show this expression for $\alpha_{z}$ which fits perfectly with the numerically obtained zigzag transition points. A similar power law dependence between the anisotropy parameter $\alpha$ and $N$ was also obtained before for 2D [18] and 3D [19] confined Coulomb clusters, using a numerical fitting procedure.

We also investigated the eigenfrequency of the center of mass mode in the $x$ and $y$ directions which could be obtained analytically. The Hamiltonian equation of motion yields

$$
\dot{v}_{x i}=-2 \alpha x_{i}+\sum_{j \neq i} \frac{x_{i}-x_{j}}{\left|\vec{r}_{i}-\vec{r}_{j}\right|^{2}}
$$

and of course we have the same for $\dot{v}_{y i}$ but with $\alpha=1$. The displacement of the center of mass $R_{x}=\Sigma_{i} x_{i}$ along the $x$ direction satisfies the differential equation

$$
\frac{d^{2} R_{x}}{d t^{2}}=\sum_{i} \dot{v}_{x_{i}}=-2 \alpha R_{x}
$$

and the same for $R_{y}$ but with $\alpha=1$. We conclude that the eigenfrequencies of the center of mass related to the directions $x$ and $y$ are, respectively, $\omega_{x}=\sqrt{2 \alpha}$ and $\omega_{y}=\sqrt{2}$. These frequencies are independent of the number of charged particles and the interparticle potential, which is predicted by the generalized Kohn theorem and is identical for quantum systems [20].

The breathing mode for $\alpha=1$ is always equal to 2 independently of the number of particles. We can demonstrate this analytically. The mean square radius $R^{2}=\sum_{i}\left(x_{i}^{2}+y_{i}^{2}\right)$ satisfies the following differential equation:

$$
\frac{d^{2} R^{2}}{d t^{2}}=2 T-4 \sum_{i=1}^{N}\left(\alpha x_{i}^{2}+y_{i}^{2}\right)+N(N-1) / 2,
$$

with $T=\sum_{i}\left(\dot{x}_{i}^{2}+\dot{y}_{i}^{2}\right)$ the total kinetic energy. In the case $\alpha=1$ the former equation reduces to

$$
\frac{d^{2} R^{2}}{d t^{2}}=2 T-4 R^{2}+N(N-1) / 2 .
$$

Thus the frequency of the breathing mode in a circular potential is $\omega=2$ independently of the number of particles.

\section{SUMMARY AND CONCLUSIONS}

The effect of the anisotropy of the confinement potential was investigated in detail for the case of particles interacting through a logarithmic potential. We show that changing the eccentricity of the confinement potential can induce first and second order transitions and that these transitions interfere in the eigenfrequencies of the system. The first order transition causes a discontinuity in almost all eigenfrequency modes while a second order transition occurs when one specific eigenfrequency becomes zero, i.e., mode softening. Also we saw that the center of mass mode is independent of the number of particles in the system and that the breathing mode in a circular confinement is always equal to 2 .

We noted that the zigzag transition is initiated by particles located in the center of the line configuration. The eigenfrequencies before the zigzag transition for a system of $N+1$ particles are the same as the eigenfrequencies of a system with $N$ particles plus two new eigenfrequencies. We presented two general expressions depending only on the eccentricity parameter and the number of particles that fit all eigenfrequencies before the zigzag transition. We obtained an analytical expression for the anisotropy parameter at which the zigzag transition occurs as a function of the number of particles in the system.

\section{ACKNOWLEDGMENTS}

This work was supported by the Flemish Science Foundation (FWO-Vl) and the Belgium Science Policy (IAP). Discussions with M. Saint Jean and A. Matulis are gratefully acknowledged.
[1] E. P. Wigner, Phys. Rev. 46, 1002 (1934).

[2] C. C. Grimes and G. Adams, Phys. Rev. Lett. 42, 795 (1979).

[3] R. C. Ashoori, Nature (London) 379, 413 (1996).

[4] M. Golosovsky, Y. Saado, and D. Davidov, Phys. Rev. E 65, 061405 (2002).
[5] J. H. Chu and L. I, Phys. Rev. Lett. 72, 4009 (1994).

[6] G. B. Hess, Phys. Rev. 161, 189 (1967); D. Stauffer and A. L. Fetter, ibid. 168, 156 (1968).

[7] Y. Pearl, Appl. Phys. Lett. 5, 65 (1964).

[8] A. I. Buzdin and J. P. Brison, Phys. Lett. A 196, 267 (1994). 
[9] L. R. E. Cabral and F. M. Peeters, Phys. Rev. B 70214522 (2004).

[10] M. Saint Jean, C. Even, and C. Guthmann, Europhys. Lett. 55, 45 (2001).

[11] M. Saint Jean and C. Guthmann, J. Phys.: Condens. Matter 14, 13653 (2002).

[12] Ying-Ju Lai and Lin I, Phys. Rev. E 60, 4743 (1999).

[13] L. J. Campbell and R. M. Ziff, Phys. Rev. B 20, 1886 (1979).

[14] V. A. Schweigert and F. M. Peeters, Phys. Rev. B 51, 7700 (1995).
[15] F. Bolton and U. Rossler, Superlattices Microstruct. 13, 139 (1993).

[16] V. M. Bedanov and F. M. Peeters, Phys. Rev. B 49, 2667 (1994).

[17] B. Partoens and P. S. Deo, Phys. Rev. B 69, 245415 (2004).

[18] L. Cândido, J. P. Rino, N. Studart, and F. M. Peeters, J. Phys.: Condens. Matter 10, 11627 (1998).

[19] J. P. Schiffer, Phys. Rev. Lett. 70, 818 (1993).

[20] F. M. Peeters, Phys. Rev. B 42, 1486 (1990). 